\title{
JVG9, a benzimidazole derivative, alters the surface and cytoskeleton of Trypanosoma cruzi bloodstream trypomastigotes
}

\author{
Dylan L Díaz-Chiguer', Francisco Hernández-Luis², Benjamín Nogueda-Torres ${ }^{3}$, Rafael Castillo², \\ Olivia Reynoso-Ducoing4, Alicia Hernández-Campos², Javier R Ambrosio ${ }^{4}+$
}
'Departamento de Investigación, Instituto de Seguridad y Servicios Sociales para los Trabajadores del Estado, Ciudad de México, México ${ }^{2}$ Departamento de Farmacia, Facultad de Química ${ }^{4}$ Departamento de Microbiología y Parasitología, Facultad de Medicina, Universidad Nacional Autónoma de México, Ciudad de México, México ${ }^{3}$ Departamento de Parasitología, Instituto Politécnico Nacional, Escuela Nacional de Ciencias Biológicas, Ciudad de México, México

Trypanosoma cruzi has a particular cytoskeleton that consists of a subpellicular network of microtubules and actin microfilaments. Therefore, it is an excellent target for the development of new anti-parasitic drugs. Benzimidazole 2-carbamates, a class of well-known broad-spectrum anthelmintics, have been shown to inhibit the in vitro growth of many protozoa. Therefore, to find efficient anti-trypanosomal (trypanocidal) drugs, our group has designed and synthesised several benzimidazole derivatives. One, named JVG9 (5-chloro-1H-benzimidazole-2-thiol), has been found to be effective against $\mathrm{T}$. cruzi bloodstream trypomastigotes under both in vitro and in vivo conditions. Here, we present the in vitro effects observed by laser scanning confocal and scanning electron microscopy on T. cruzi trypomastigotes. Changes in the surface and the distribution of the cytoskeletal proteins are consistent with the hypothesis that the trypanocidal activity of JVG9 involves the cytoskeleton as a target.

Key words: Trypanosoma cruzi - benzimidazole - cytoskeleton - tubulin - actin

Trypanosoma cruzi, an haemoflagelate protozoa, is causative of Chagas disease, a neglected tropical disease (WHO 2010) that is frequently encountered in people living in underdeveloped countries. The medical drug treatment currently available for Chagas disease is nifurtimox (Lampit ${ }^{\mathrm{TM}}$, Bayer) and benznidazole (Rochagan $^{\mathrm{TM}}$, Roche); however, both drugs have limited efficacy on chronic stages and high toxicity (Chung et al. 2003). Additionally, there are different susceptibilities to those drugs among $T$. cruzi strains, which vary according to the geographical area (Laurent \& Swindle 2000). Therefore, there is an urgent need for the research and development of new drugs against $T$. cruzi.

The cytoskeleton of T. cruzi parasites is primarily composed of a subpellicular corset of microtubules (Gull 1999) whose structure is vital for the shape, form, motility and survival/infectivity of the parasites. Actin is an important cytoskeletal protein that is present in all stages of T. cruzi, as demonstrated by Cevallos et al. (2011), on the body, flagellum and flagellar pocket regions. Therefore, any alteration induced by the effects of cytoskeletal disrupting agents, as found for benzimidazole chemicals, has a clear effect on the morphology of the parasites. JVG9 (5-chloro-1 $H$-benzimidazole-2-thiol), an

doi: 10.1590/0074-0276140096

Financial support: DGAPA-UNAM (IN201510, IN216213),

DGAPA-IXTLI-UNAM (IX200610)

+ Corresponding author: jrah@unam.mx

Received 17 March 2014

Accepted 11 July 2014 experimental benzimidazole derivative, has been shown to produce both trypanocidal effects against $T$. cruzi bloodstream trypomastigotes and to have low cellular toxicity compared with nifurtimox and other benzimidazole drugs (Díaz-Chiguer et al. 2012). These effects are notable because of the well-recognised anthelmintic activity of benzimidazole-2-carbamates (Valdez et al. 2002). In other studies, these chemicals inhibited the in vitro growth of the protozoa Giardia lamblia (Cedillo \& Muñoz 1992) and Leishmania infantum (Armson et al. 1999). Benzimidazole appears to interfere with microtubule dynamics by binding to $\beta$-tubulin (Lacey 1988) and there is evidence that these derivatives can inhibit the in vitro growth of some protozoa (Katiyar et al. 1994). Therefore, because these chemicals are potential antiprotozoa agents, our group has designed and synthesised a new series of $1 H$-benzimidazole derivatives and tested them against $G$. lamblia, Entamoeba histolytica and Trichinella spiralis to increase their efficacy (Valdez et al. 2002). One of these compounds, the derivative JVG9, was found to be effective against $T$. cruzi bloodstream trypomastigotes (Díaz-Chiguer et al. 2012); until now, however, its effects on the morphology and organisation of the parasite cytoskeleton have not been evaluated. We demonstrate in the present paper that JVG9 has a clear effect on the morphology of T. cruzi bloodstream trypomastigotes and produces alterations in the expression of the cytoskeletal proteins actin and tubulin.

\section{MATERIALS AND METHODS}

Compound - JVG9 was prepared as described previously. It was purified by column chromatography on silica gel and its chemical structure was confirmed by IR, 1H-NMR and MS data (Navarrete-Vázquez et al. 2001, Valdez et al. 2002). 
Parasites - T. cruzi bloodstream trypomastigotes INC-5 strain (MHOM/MX/97/Inc-5) corresponding to the lineage I (Bosseno et al. 2002) were obtained via heart puncture of T. cruzi-infected NIH female, as described by Díaz-Chiguer et al. (2012). Animal experiments were performed according to Official Mexican Standards (NOM-062-ZOO-1999).

In vitro effects of JVG9 on parasites - The effects on T. cruzi bloodstream trypomastigotes were evaluated as described previously (Díaz-Chiguer et al. 2012). Briefly, bloodstream trypomastigote forms were incubated in $54.2 \mu \mathrm{m}$ JVG9 diluted in dimethyl sulfoxide (DMSO) for $24 \mathrm{~h}$. The final concentration of DMSO in the culture medium remained below $1 \%$. The parasites were then removed and processed for scanning electron microscopy (SEM) and laser scanning confocal microscopy (LSCM). Parasites cultivated in the presence of DMSO $(<1 \%)$ were considered negative controls.

SEM - Parasites were processed as previously described (Márquez-Navarro et al. 2013). Briefly, parasites were fixed with $2.5 \%$ glutaraldehyde in $0.1 \mathrm{M}$ sodium cacodilate ( $\mathrm{pH}$ 7.2), subsequently washed three times with phosphate buffered saline (PBS), post-fixed with $2 \%$ osmium tetroxide (EM grade), dehydrated through a graded ethanol series, dried in a critical point drier and coated with ionised gold (15 nm thick). Images using taken with a Zeiss DSM-950 microscope adjusted to $15 \mathrm{kV}$. Image contrast was performed using the Adobe Photoshop v.8.0 software.

Immunofluorescence assays - Parasites were prepared as previously described (Valverde-Islas et al. 2011) with slight modifications. Briefly, after parasites were washed with sterile buffer-PBS, they were placed onto poly-Llysine coated coverslips. The samples were then fixed with $4 \%$ paraformaldehyde $(\mathrm{w} / \mathrm{v})$, permeabilised with $0.3 \%$ (v/v) Tween-20, blocked with $2 \%(\mathrm{w} / \mathrm{v})$ bovine serum albumin in PBS and incubated overnight at $4^{\circ} \mathrm{C}$ with a primary anti- $\alpha$-tubulin antibody DM1A (1:100) (Santa Cruz Biotechnology Inc, USA) with fluorescein isothiocyanate-conjugated anti-mouse IgG (1:200) (ZYMED, USA) as a secondary antibody. Actin was revealed by an indirect immunoassay in which the primary antibody was a polyclonal anti-T. cruzi antibody (1:200) (Cevallos et al. 2011) and the secondary antibody was an anti-rabbit antibody coupled to biotin (1:30), followed by incubation with rhodamine-conjugated avidin (1:150) (Vector Laboratories, USA). DNA was stained with 4',6-diamidino-2phenylindole $\left(\operatorname{Sigma}^{\circledR}\right)(1 \mathrm{mg} / \mathrm{mL})$. Samples were mounted in mounting medium $\left(\mathrm{DAKO}^{\circledR}\right)$ and observed using a LSCM (TCS-MP5) Leica equipped with ultraviolet light, argon and helium-neon lasers and an objective lens (HCX PL APO lambda blue $63.0 \times 1.40$ OIL UV).

\section{RESULTS}

With SEM, untreated bloodstream trypomastigotes appear to present a typical morphology of flagella, undulating membranes and a homogeneous surface (Fig. 1A, B). After treatment with JVG9, parasites were found to have important alterations in their topography, with a decreased flagellar size and, in several parasites, disruptions at their posterior ends (Fig. 1C-E). Additionally, several membrane protrusions appeared as blebs at the level of their cytoplasmic membranes (Fig. 1F).

Using the technique of LSCM (Fig. 2), JVG9 was found to restructure actin and $\alpha$-tubulin in $T$. cruzi trypomastigotes. Untreated parasites (Fig. 2) presented a different distribution of these cytoskeletal proteins in both their cellular bodies and flagella. The fluorescence pattern of actin was homogeneously distributed in parasites
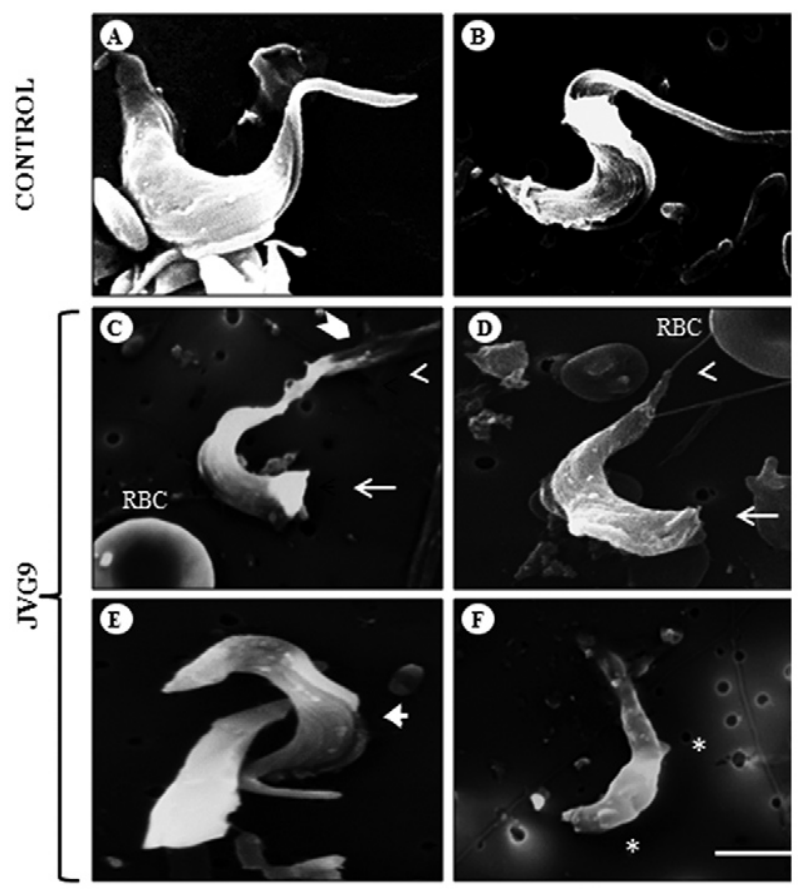

Fig. 1: scanning electron microscopy of bloodstream forms of Trypanosoma cruzi INC-5 strain. Control parasites showing typical morphology of the body and flagellum (A, B). Treatment of the parasites with $54.2 \mu \mathrm{m}$ JVG9 during $24 \mathrm{~h}(\mathrm{C}-\mathrm{F})$ induced the formation of blebs (asterisk), alterations in the anterior (open arrowhead) and posterior (arrow) ends, disruption of the flagellum (chevron symbol) and membrane undulating (arrowhead). Bar $=5 \mu \mathrm{m}$. RBC: red blood cell.

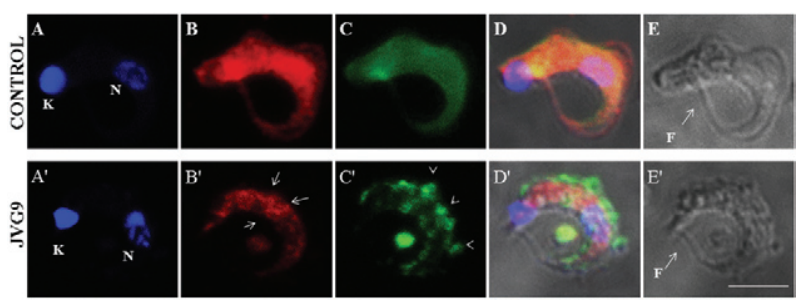

Fig. 2: laser scanning confocal microscopy images of bloodstream forms of Trypanosoma cruzi INC-5 strain. Upper panel: fluorescent staining of control parasite with DAPI (A), actin of T. cruzi (B), $\alpha$-tubulin (C) MERGE (D) and Nomarsky illumination (E); bottom panel: bloodstream forms of $T$. cruzi INC-5 strain treated with JVG9 compound $54.2 \mu \mathrm{m}$ during $24 \mathrm{~h}$. The actin patches are indicating by arrows and clusters of $\alpha$-tubulin are indicated by open arrowheads. Bar $=5 \mu \mathrm{m}$. F: flagellum; K: kinetoplast; N: nucleus. 
of the control group (Fig. 2B) whereas in treated parasites the protein was found to be aggregated in patches on the body, but not in the flagellum (Fig. 2B), and $\alpha$-tubulin was distributed in clusters that were located primarily at the periphery (Fig. 2C). Morphological changes have a strong correlation with the distribution of the microfilaments and microtubules in bloodstream trypomastigotes.

\section{DISCUSSION}

Because Chagas disease is still considered a public health problem and a neglected disease in many underdevelopment countries, the efforts to find effective drugs for its treatment continue. Many experimental compounds have been designed, produced and tested against T. cru$z i$, but many of them remain unused until their impact in the biology or the physiology of the parasite can be established. It is well known that the application of different approaches to the study of the cellular biology of the parasites has a great influence on drug development (Coura \& de Castro 2002, Paulino et al. 2005, Romero \& Morilla 2010, Urbina 2010). After the demonstration that the JVG9 is a benzimidazole derivative with trypanocidal activity (Díaz-Chiguer et al. 2012), we here demonstrated that this compound produces alterations in the morphology and distribution of the cytoskeletal proteins actin and tubulin in bloodstream forms of the INC-5 strain. However, we noted that the concentration, at which the effects were observed in parasites, was six times lower than the LC50 reported in our previous work (Díaz-Chiguer et al. 2012). We therefore decided to treat the parasites at 54.2 $\mu \mathrm{m}$ because this was the concentration at which we first observed an effect on cytoskeletal proteins such as actin and tubulin. Alternately, at higher concentrations, such as $320 \mu \mathrm{m}$, it was not possible to record any microscopic observations due the presence of precipitation.

It is possible that observed alterations on the parasite cytoskeletal proteins that produced changes in the morphology may be similar to those found after anti-cancer treatment. When Leishmania amazonensis and T. cruzi were evaluated with taxol and vinblastine, effects were clearly found on the membrane, with protrusions that resemble cellular surface blebs indicative of cell death mechanisms such as mitochondrial swelling, plasma membrane blebbing and the presence of autophagosomelike structures (Vannier-Santos \& de Castro 2009). If JVG9 is capable of triggering some form of this parasite death mechanism, this would be the first report of the involvement of alterations in the expression of cytoskeletal proteins in the presence of a benzimidazole-derivative compound. It would be worthwhile to evaluate the expression of cytoskeletal proteins using double-dimension electrophoresis, as published previously for actin (Cevallos et al. 2011) and to determine whether there are changes in the expression of actin and tubulin variants and whether they are the primary targets of these compounds. The effects of the designed JVG9 are a very good example of a structure-activity integration that produces better drugs through medicinal chemistry approaches. Additionally, it is an alternative approach showing how it is possible to redesign old drugs and thereby find novel compounds for the treatment of Chagas disease.

\section{ACKNOWLEDGEMENTS}

To Dr Adrián Márquez-Navarro, Dr Laura Valverde-Islas CD, María José Gómora Herrera and the biologist Armando Zepeda-Rodríguez, for their general technical assistance, and to Dr Ana María Cevallos Gaos, for the gift of the anti-actin antibody of $T$. cruzi.

\section{REFERENCES}

Armson A, Kamau SW, Grimm F, Reynoldson JA, Best WM, MacDonald LM, Thompson RC 1999. A comparison of the effects of a benzimidazole and the dinitroanilines against Leishmania infantum. Acta Trop 73: 303-311.

Bosseno MF, Barnabe C, Gastelum EM, Kasten FL, Ramsey J, Espinoza B, Breniere SF 2002. Predominance of Trypanosoma cruzi lineage I in Mexico. J Clin Microbiol 40: 627-632.

Cedillo RR, Muñoz O 1992. In vitro susceptibility of Giardia lamblia to albendazole, mebendazole and other chemotherapeutic agents. J Med Microbiol 37: 221-223.

Cevallos AM, Segura-Kato YX, Merchant-Larios H, Manning-Cela R, Hernández-Osorio LA, Márquez-Duenas C, Ambrosio JR, Reynoso-Ducoing O, Hernández R 2011. Trypanosoma cruzi: multiple actin isovariants are observed along different developmental stages. Exp Parasitol 127: 249-259.

Chung MC, Guido RV, Martinelli TF, Goncalves MF, Polli MC, Botelho KC, Varanda EA, Colli W, Miranda MT, Ferreira EI 2003. Synthesis and in vitro evaluation of potential antichagasic hydroxymethylnitrofurazone (NFOH-121): a new nitrofurazone prodrug. Bioorg Med Chem 11: 4779-4783.

Coura JR, de Castro SL 2002. A critical review on Chagas disease chemotherapy. Mem Inst Oswaldo Cruz 97: 3-24.

Díaz-Chiguer DL, Márquez-Navarro A, Nogueda-Torres B, de la Luz LAG, Pérez-Villanueva J, Hernández-Campos A, Castillo R, Ambrosio JR, Nieto-Meneses R, Yépez-Mulia L, HernándezLuis F 2012. In vitro and in vivo trypanocidal activity of some benzimidazole derivatives against two strains of Trypanosoma cruzi. Acta Trop 122: 108-112.

Gull K 1999. The cytoskeleton of trypanosomatid parasites. Annu Rev Microbiol 53: 629-655.

Katiyar SK, Gordon VR, McLaughlin GL, Edlind TD 1994. Antiprotozoal activities of benzimidazoles and correlations with betatubulin sequence. Antimicrob Agents Chemother 38: 2086-2090.

Lacey E 1988. The role of the cytoskeletal protein, tubulin, in the mode of action and mechanism of drug resistance to benzimidazoles. Int J Parasitol 18: 885-936.

Laurent JP, Swindle J 2000. Trypanosoma cruzi: sensitivity to selective drugs in phylogenetically unrelated stocks. Exp Parasitol 94: 60-61.

Márquez-Navarro A, Pérez-Reyes A, Zepeda-Rodríguez A, ReynosoDucoing O, Hernández-Campos A, Hernández-Luis F, Castillo R, Yépez-Mulia L, Ambrosio JR 2013. RCB20, an experimental benzimidazole derivative, affects tubulin expression and induces gross anatomical changes in Taenia crassiceps cysticerci. Parasitol Res 112: 2215-2226.

Navarrete-Vázquez G, Cedillo R, Hernández-Campos A, Yépez L, Hernández-Luis F, Valdez J, Morales R, Cortés R, Hernández M, Castillo R 2001. Synthesis and antiparasitic activity of 2-(trifluoromethyl)-benzimidazole derivatives. Bioorg Med Chem Lett 11: 187-190.

Paulino M, Iribarne F, Dubin M, Aguilera-Morales S, Tapia O, Stoppani AO 2005. The chemotherapy of Chagas disease: an overview. Mini Rev Med Chem 5: 499-519. 
Romero EL, Morilla MJ 2010. Nanotechnological approaches against Chagas disease. Adv Drug Deliv Rev 62: 576-588.

Urbina JA 2010. Specific chemotherapy of Chagas disease: relevance, current limitations and new approaches. Acta Trop 115: 55-68.

Valdez J, Cedillo R, Hernández CA, Yépez L, Hernández LF, Navarrete VG, Tapia A, Cortés R, Hernández M, Castillo R 2002. Synthesis and antiparasitic activity of $1 \mathrm{H}$-benzimidazole derivatives. Bioorg Med Chem Lett 12: 2221-2224.
Valverde-Islas LE, Arrangoiz E, Vega E, Robert L, Villanueva R, Reynoso-Ducoing O, Willms K, Zepeda-Rodríguez A, Fortoul TI, Ambrosio JR 2011. Visualization and 3D reconstruction of flame cells of Taenia solium (cestoda). PLoS ONE 6: e14754.

Vannier-Santos MA, de Castro SL 2009. Electron microscopy in antiparasitic chemotherapy: a (close) view to a kill. Curr Drug Targets 10: $246-260$

WHO - World Health Organization 2010. The 17 neglected tropical diseases. Available from: who.int/neglected_diseases/diseases/en/. 